\title{
Comparação entre métodos de estimativa da evapotranspiração de referência (ETo) na região Norte Fluminense, $\mathrm{RJ}^{1}$
}

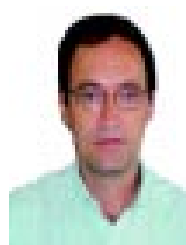

\author{
José C. Mendonça ${ }^{2}$, Elias F. de Sousa ${ }^{3}$, Salassier Bernardo ${ }^{4}$, Gutemberg P. Dias ${ }^{5}$ \& Sidney Grippa ${ }^{6}$ \\ 1 Trabalho realizado com apoio da SEAP, FENORTE, FAPERJ, CAPES, CNPq e da PESAGRO/Rio \\ 2 Laboratório de Engenharia Agrícola/CCTA/UENF. Av. Alberto Lamego 2000, Horto, CEP 28015-620, Campos dos \\ Goytacazes, RJ. Fone: (22) 2726-1543. E-mail: mendonca@uenf.br (Foto) \\ 3 UENF/CCTA/LEAG. E-mail: efs@uenf.br \\ 4 UENF/CCTA/LEAG. E-mail: salassie@uenf.br \\ ${ }^{5}$ DEA/UFV. CEP 36571-000, Viçosa, MG. E-mail: gpdias@ufv.br \\ 6 UENF/CCTA/LEAG
}

Protocolo 195 - 30/12/2002 - Aprovado em 28/8/2003

\begin{abstract}
Resumo: Este estudo foi desenvolvido na Estação Evapotranspirométrica da UENF, instalada na área da Estação Experimental da PESAGRO-Rio, em Campos dos Goytacazes, RJ. Um lisímetro de pesagem com $6 \mathrm{~m}^{2}$ de área, dotado de células de carga, foi instalado no centro de uma área de $900 \mathrm{~m}^{2}$, cultivada com grama tipo Batatais ( $P$. notatum L.). O objetivo deste estudo foi propor coeficientes de ajuste regional entre o método lisimétrico e os métodos de Penman-MonteithFAO, Makkink, Linacre, Jensen-Haise, Hargreaves-Samani, Radiação Solar, Tanque Classe "A" e Atmômetro SEEl modificado para os períodos de 1, 3, 7 e 10 dias. Os resultados obtidos indicam que, com exceção do Atmômetro, todos os métodos avaliados atendem satisfatoriamente à estimativa da ETo na região Norte Fluminense do Estado do Rio de Janeiro, apresentando bons ajustamentos para períodos maiores que sete dias.
\end{abstract}

Palavras-chave: evapotranspiração, lisímetro, consumo de água

\section{Comparison of estimation methods of reference crop evapotranspiration (ETo) for Northeren Region of Rio de Janeiro State, Brazil}

\begin{abstract}
The aim of this research paper was to make a comparison between the reference values of crop evapotraspiration acquired through the lysimeter and the FAO-modified PenmanMonteith, Solar Radiation, Makkink, Linacre, Jensen-Haise, Hargreaves-Samani, PAN "Class A" and modified SEEI Atmometer methods. Data was obtained from the meteorological station at PESAGRO-Rio, located at Campos dos Goytacazes in the State of Rio de Janeiro State, Brazil. Results have shown that for the northern region of Rio de Janeiro, the methods tend to be well adjusted to the reference values of the crop evapotranspiration as to those obtained through the lysimeter. Nonetheless, good adjustments were obtained for intervals longer than seven-day periods when using the indirect and evaporimeter methods.
\end{abstract}

Key words: evapotranspiration, lysimeter, water consumption

\section{INTRODUÇÃO}

A determinação da quantidade de água necessária para as culturas é um dos principais parâmetros para o correto planejamento, dimensionamento e manejo de qualquer sistema de irrigação. Sua quantificação é realizada fazendo-se o balanço hídrico da camada do solo ocupada pelo sistema radicular da cultura, o qual tem, na "evapotranspiração" e na precipitação pluviométrica, seus principais componentes (Bernado, 1995). "Evapotranspiração" foi o termo utilizado por Thornthwaite no início da década de 40 do século passado, para expressar esta ocorrência simultânea dos processos de evaporação da água no solo e da transpiração das plantas. Ela é controlada pelo balanço de energia, pela demanda atmosférica e pelo suprimento de água do solo às plantas. Pereira et al. (1997), definem a evapotranspiração como um elemento climatológico fundamental, que corresponde ao processo oposto da chuva, também expressa em milímetros.

O termo evapotranspiração de referência (ETo) foi definido por Doorenbos \& Pruitt (1977) como aquela que ocorre em uma extensa superfície coberta com grama de 0,08 a $0,15 \mathrm{~m}$, em crescimento ativo, cobrindo totalmente o solo e sem deficiência de água. Em 1991, pesquisadores de vários países, especialistas em agrometeorologia, concluíram que o conceito de ETo 
empregado apresentava problemas, sobretudo relacionados à variabilidade das culturas de referência atualmente em uso, levando a erros de sub ou superestimações e à existência de dificuldades experimentais para contrastá-los. Essas condições dificultavam a determinação padronizada da ETo em grande escala.

Bernado et al. (1996) relatam que a ETo pode ser determinada por métodos diretos e indiretos, sendo os métodos diretos os que utilizam lisímetros, parcelas experimentais no campo, controle de umidade do solo e método de entrada e saída de água em grandes áreas. Dos métodos diretos, o procedimento mais preciso para se determinar a ETo é a utilização de lisímetros. Por apresentarem custos elevados, seu uso tem ficado restrito a instituições de pesquisas, tendo sua utilização justificada na calibração regional de métodos indiretos.

Diversos pesquisadores em todo o mundo propuseram modelos indiretos para a estimativa da ETo, com as mais diferentes concepções e número de variáveis envolvidas. Antes de se eleger o modelo a ser utilizado para a estimativa da ETo, é necessário saber quais os elementos climáticos disponíveis; a partir daí, verifica-se quais podem ser aplicados, uma vez que a utilização dos diferentes métodos para certo local de interesse fica na dependência dessas variáveis.

O objetivo deste trabalho foi comparar os valores de ETo obtidos em lisímetro de pesagem com grama com os valores resultantes da utilização dos métodos de Penman-Monteith parametrizado pela FAO, Radiação Solar, Makkink, Linacre, Jensen-Haise, Hargreaves-Samani, Tanque Classe "A" e Atmômetro SEEI modificado.

\section{MATERIAL E MÉTODOS}

Os trabalhos foram realizados na área da Estação Evapotranspirométrica da UENF/PESAGRO-Rio, localizada na Estação Experimental de Campos dos Goytacazes, RJ. Utilizou-se tanque lisimétrico com dimensões de 3,0 x 2,0 x 1,5 m, confeccionado em chapa metálica. A pesagem era realizada por um conjunto de 4 células de carga fabricadas pela J-Star Electronics, Wisconsin, EUA, e instaladas na base do tanque.

Os dados diários de temperatura do ar, radiação solar, umidade relativa, pressão atmosférica e velocidade do vento, foram coletados por uma estação eletrônica automática, fabricada pela Thies Clima. A determinação da lâmina evapotranspirada pelo lisímetro foi obtida pela variação do peso observado no período, dividido pela área da superfície evaporante $\left(6 \mathrm{~m}^{2}\right)$. Para o cálculo da ETo pelo método parametrizado pela FAO, utilizou-se a metodologia apresentada por Mello (1998) e os métodos Radiação Solar, Makkink, Linacre, Jensen-Haise, Hargreaves-Samani e Tanque Classe A, foram desenvolvidos segundo metodologia proposta por Pereira et al. (1997). O cálculo da ETo pelo método do Atmômetro SEEI modificado foi obtido diretamente pelas diferenças entre as leituras diárias do nível da água localizada na parte externa do aparelho.

Para as correlações dos valores da ETo medidos no lisímetro de pesagem com os estimados pelos métodos indiretos e evaporímetros, para o período de 1 dia, entre os meses de setembro de 1997 a dezembro de 2000, buscou-se eliminar os valores obtidos em dias em que ocorreram precipitação, irrigação, poda do gramado e dias de eventuais anormalidades, tais como falta de energia elétrica e necessidade de manutenção dos equipamentos. Para correlações dos valores em períodos de 3, 7 e 10 dias, realizou-se o agrupamento das variáveis meteorológicas componentes de cada método, com os valores selecionados no lisímetro de pesagem, procedendo-se à composição das médias móveis das variáveis para os períodos estudados.

Os valores de ETo utilizados neste trabalho constam de uma série de 680 observações obtidas pelos métodos indiretos e Tanque Classe "A", e 353 observações, pelo método do Atmômetro SEEI modificado.

\section{RESULTADOS E DISCUSSÃO}

A Tabela 1 apresenta as médias mensais da ETo determinadas através dos métodos avaliados, na qual se verifica que os métodos de Penman-Monteith FAO, Radiação Solar, Linacre, Jensen-Haise, Hargreaves-Samani e Tanque Classe "A", apresentaram tendências de superestimar a ETo na região Norte Fluminense, RJ . Esses métodos superestimaram a ETo em 9,7, $25,7,15,9,51,3,23,5$ e $30 \%$, respectivamente.

Os métodos Radiação Solar, Jensen-Haise, HargreavesSamani e Tanque Classe "A", superestimaram o lisímetro em

Tabela 1. Variação dos valores médios mensais da ETo determinada pelo método do lisímetro de pesagem (LP) e pelos métodos* comparados, em mm dia-1

\begin{tabular}{|c|c|c|c|c|c|c|c|c|c|}
\hline \multirow{2}{*}{ Mês } & \multicolumn{9}{|c|}{ Método } \\
\hline & LP & $\mathrm{PM}$ & MAK & LIN & $\mathrm{J}-\mathrm{H}$ & $\mathrm{H}-\mathrm{S}$ & $\mathrm{RS}$ & TCA & ATM \\
\hline Janeiro & 4,6 & 5,2 & 4,5 & 4,5 & 7,5 & 5,8 & 6,0 & 6,0 & 2,9 \\
\hline Fevereiro & 5,4 & 5,1 & 4,4 & 4,5 & 7,4 & 5,5 & 5,9 & 5,9 & 2,6 \\
\hline Abril & 3,4 & 3,4 & 3,0 & 3,9 & 4,9 & 3,8 & 3,9 & 4,0 & 1,9 \\
\hline Maio & 2,5 & 2,6 & 2,4 & 3,5 & 3,6 & 3,1 & 3,0 & 3,3 & 1,7 \\
\hline Junho & 2,9 & 2,8 & 2,5 & 3,4 & 3,8 & 3,3 & 2,7 & 2,7 & 1,5 \\
\hline Setembro & 3,3 & 4,0 & 3,4 & 3,7 & 5,3 & 4,1 & 4,5 & 4,6 & 1,9 \\
\hline Outubro & 3,6 & 4,0 & 3,3 & 3,8 & 5,1 & 4,5 & 4,5 & 4,6 & 1,8 \\
\hline Novembro & 3,4 & 4,2 & 3,6 & 3,7 & 5,7 & 4,7 & 4,7 & 4,9 & 2,3 \\
\hline Dezembro & 4,5 & 5,0 & 4,3 & 4,2 & 7,1 & 5,4 & 6,0 & 5,9 & 2,5 \\
\hline ETo média & 3,5 & 3,8 & 3,3 & 3,8 & 5,3 & 4,2 & 4,4 & 4,5 & 2,1 \\
\hline
\end{tabular}

"PM: Penman-Monteith-FAO; MAK: Makkink; LIN: Linacre; J_H: Jensen-Haise; H-S: Hargreaves-Samani; RS: Radiação Solar; TCA: Tanque Classe "A" e ATM: Atmômetro SEEI modificado 
todos os meses do ano, e os métodos de Makkink e Atmômetro subestimaram a ETo determinada no lisímetro em 4,2 e 38,3\% respectivamente. Em valores médios ocorridos no período do estudo, o método de Makkink foi o que mais se aproximou do valor medido no lisímetro, seguido pelo método de PenmanMonteith-FAO.

Na Tabela 2 tem-se os coeficientes de regressão, o coeficiente de determinação $\left(\mathrm{r}^{2}\right)$ e o erro padrão de estimativa (EPE) obtidos do ajustamento linear entre os valores estimados pelos métodos indiretos e evaporímetros e os medidos pelo lisímetro de pesagem, para os períodos de 1, 3, 7 e 10 dias. As Figuras de 1 a 4 apresentam os gráficos resultantes da regressão linear, respectivamente para os períodos de 1, 3, 7 e 10 dias.

Tabela 2. Parâmetros estatísticos da regressão linear $(y=a+b x)$ entre os valores da ETo medida pelo lisímetro de pesagem (LP) e as determinadas pelos métodos comparados

\begin{tabular}{|c|c|c|c|c|}
\hline Intervalo (Dias) & $\mathrm{a}$ & B & $r^{2}$ & $\mathrm{EPE}^{* *}$ \\
\hline & \multicolumn{4}{|c|}{ Penman-Monteith-FAO } \\
\hline 1 & $0,08 \pm 0,12$ & $0,88 \pm 0,02$ & 0,58 & 1,00 \\
\hline 3 & $-0,10 \pm 0,11$ & $0,94 \pm 0,02$ & 0,74 & 0,65 \\
\hline 7 & $-0,18 \pm 0,11$ & $0,96 \pm 0,02$ & 0,80 & 0,53 \\
\hline \multirow[t]{2}{*}{10} & $-0,20 \pm 0,10$ & $0,97 \pm 0,02$ & 0,82 & 0,49 \\
\hline & \multicolumn{4}{|c|}{ Makkink $^{*}$} \\
\hline 1 & $0,34 \pm 0,11$ & $0,94 \pm 0,03$ & 0,54 & 1,05 \\
\hline 3 & $0,00 \pm 0,10$ & $1,04 \pm 0,02$ & 0,70 & 0,70 \\
\hline 7 & $-0,25 \pm 0,10$ & $1,12 \pm 0,02$ & 0,77 & 0,57 \\
\hline \multirow[t]{2}{*}{10} & $-0,30 \pm 0,10$ & $1,14 \pm 0,02$ & 0,79 & 0,53 \\
\hline & \multicolumn{4}{|c|}{ Linacre } \\
\hline 1 & $-3,54 \pm 0,08$ & $1,83 \pm 0,01$ & 0,48 & 1,12 \\
\hline 3 & $-4,28 \pm 0,08$ & $2,03 \pm 0,01$ & 0,69 & 0,72 \\
\hline 7 & $-4,72 \pm 0,08$ & $2,15 \pm 0,01$ & 0,79 & 0,55 \\
\hline \multirow[t]{2}{*}{10} & $-4,87 \pm 0,08$ & $2,19 \pm 0,01$ & 0,82 & 0,53 \\
\hline & \multicolumn{4}{|c|}{ Jensen-Haise $^{*}$} \\
\hline 1 & $0,51 \pm 0,16$ & $0,55 \pm 0,02$ & 0,57 & 1,01 \\
\hline 3 & $0,31 \pm 0,15$ & $0,60 \pm 0,02$ & 0,73 & 0,69 \\
\hline 7 & $0,16 \pm 0,14$ & $0,63 \pm 0,02$ & 0,80 & 0,54 \\
\hline \multirow[t]{2}{*}{10} & $0,12 \pm 0,14$ & $0,64 \pm 0,02$ & 0,82 & 0,49 \\
\hline & \multicolumn{4}{|c|}{ Hargreaves-Samani ${ }^{*}$} \\
\hline 1 & $-0,34 \pm 0,11$ & $0,90 \pm 0,02$ & 0,49 & 1,11 \\
\hline 3 & $-0,61 \pm 0,11$ & $0,96 \pm 0,02$ & 0,67 & 0,74 \\
\hline 7 & $-0,76 \pm 0,11$ & $1,00 \pm 0,02$ & 0,76 & 0,58 \\
\hline \multirow[t]{2}{*}{10} & $-0,78 \pm 0,11$ & $1,00 \pm 0,02$ & 0,79 & 0,53 \\
\hline & \multicolumn{4}{|c|}{ Radiação Solar-Fao ${ }^{*}$} \\
\hline 1 & $0,56 \pm 0,14$ & $0,66 \pm 0,03$ & 0,54 & 1,05 \\
\hline 3 & $0,22 \pm 0,13$ & $0,74 \pm 0,02$ & 0,70 & 0,70 \\
\hline 7 & $-0,02 \pm 0,12$ & $0,80 \pm 0,02$ & 0,78 & 0,57 \\
\hline \multirow[t]{2}{*}{10} & $-0,10 \pm 0,12$ & $0,82 \pm 0,02$ & 0,80 & 0,53 \\
\hline & \multicolumn{4}{|c|}{ Tanque "Classe A"* } \\
\hline 1 & $0,77 \pm 0,14$ & $0,59 \pm 0,02$ & 0,43 & 1,15 \\
\hline 3 & $0,35 \pm 0,12$ & $0,69 \pm 0,02$ & 0,56 & 0,85 \\
\hline 7 & $0,08 \pm 0,12$ & $0,75 \pm 0,02$ & 0,63 & 0,73 \\
\hline \multirow[t]{2}{*}{10} & $-0,02 \pm 0,11$ & $0,77 \pm 0,02$ & 0,66 & 0,68 \\
\hline & \multicolumn{4}{|c|}{ Atmômetro Seei Modificado ${ }^{*}$} \\
\hline 1 & $1,18 \pm 0,10$ & $0,84 \pm 0,03$ & 0,32 & 1,43 \\
\hline 3 & $0,39 \pm 0,09$ & $1,21 \pm 0,03$ & 0,52 & 1,11 \\
\hline 7 & $-0,12 \pm 0,09$ & $1,45 \pm 0,02$ & 0,66 & 1,01 \\
\hline 10 & $-0,21 \pm 0,09$ & $1,50 \pm 0,02$ & 0,71 & 0,98 \\
\hline
\end{tabular}

* Significativos a nível de $1 \%$ de probabilidade pelo Teste

* Erro Padrão de Estimativa
Penman Monteith-FAO

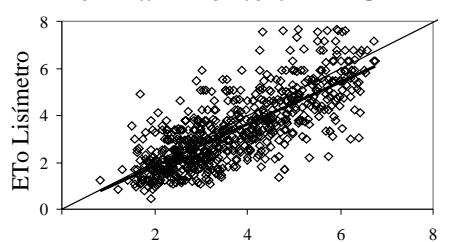

Linacre

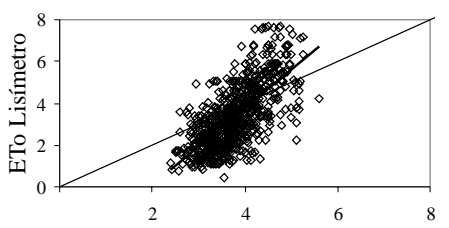

Hargreaves-Samani

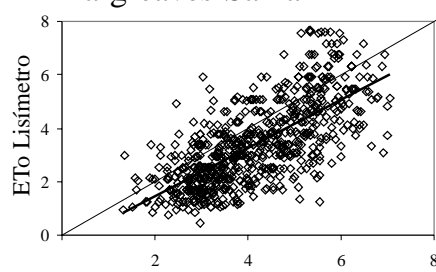

Tanque "Classe A"

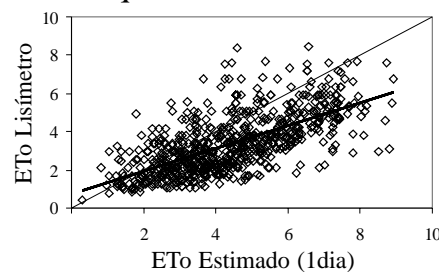

Makkink

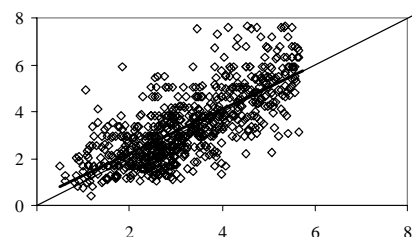

Jensen-Haise

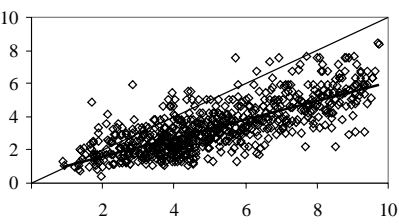

Radiação Solar-FAO

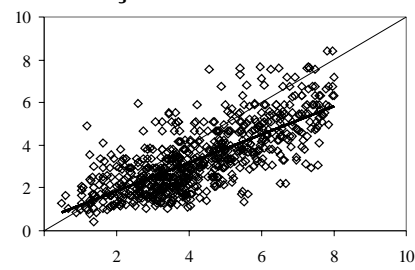

Atmômetro SEEI Modificado

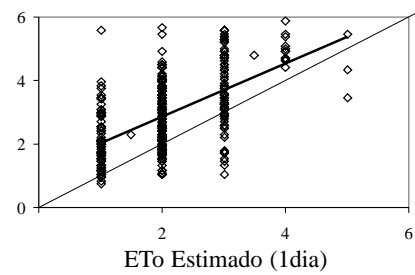

Figura 1. Correlações entre os valores da ETo medidos e estimados, pelos métodos avaliados, $\mathrm{em} \mathrm{mm} \mathrm{dia}^{-1}$, no período de 1 dia, na região Norte Fluminense, RJ

Observa-se, na mesma tabela e figuras, que as estimativas da ETo apresentaram, pelos métodos indiretos, um bom ajustamento com os valores medidos, notadamente nos períodos de 7 e 10 dias, o mesmo não ocorrendo com os evaporímetros, que não apresentaram ajustamentos satisfatórios. No período de 1 dia, percebem-se os menores ajustamentos, comportamento também observado por Peres et al. (1995), que o atribuíram à inércia apresentada pelos lisímetros em relação à sua drenagem, caracterizando retenção da água livre no solo, viciando as informações coletadas.

Observa-se ainda aumento progressivo dos coeficientes de determinação com o aumento do período de observação apresentando, para períodos decendiais, coeficientes de determinação $\left(\mathrm{r}^{2}\right)$ de $0,82,0,80,0,79,0,82,0,82,0,79,0,66$ e 0,71 e erro padrão de estimativa (EPE) de $0,49,0,53,0,53,0,53,0,49$, 0,53, 0,68 e 0,98, respectivamente para os métodos de PenmanMonteith-FAO, Radiação Solar, Makkink, Linacre, JensenHaise, Hargreaves-Samani, Tanque Classe "A" e Atmômetro SEEI modificado, atribuindo a justificativa desse desempenho, em parte, à suavização no comportamento das variáveis meteorológicas promovidas pelo uso da média móvel.

Os menores valores observados nos coeficientes de determinação $\left(\mathrm{r}^{2}\right)$ foram obtidos com o método do Tanque Classe "A" e do Atmômetro, 0,43 e 0,32, respectivamente, para o período de 1 dia. Observa-se ainda que o método de PenmanMonteith apresentou o melhor desempenho comparativamente 


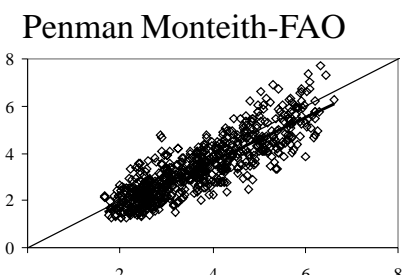

Hargreaves-Samani

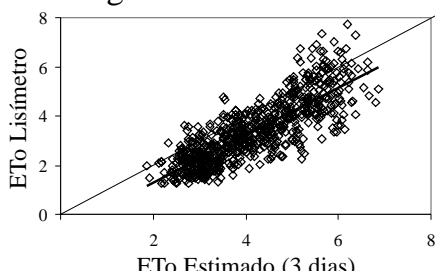

Makkink

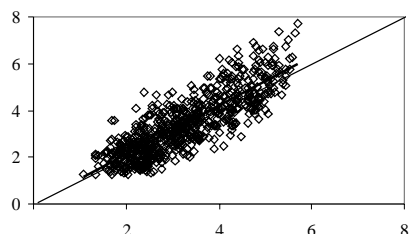

Radiação Solar-FAO

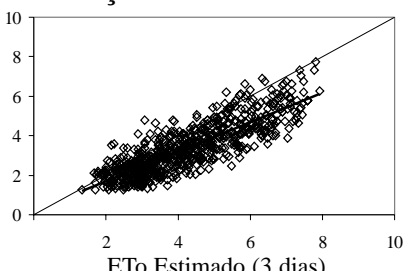

Linacre

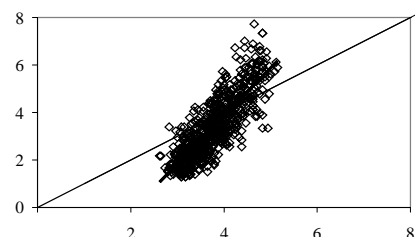

Tanque "Classe A"

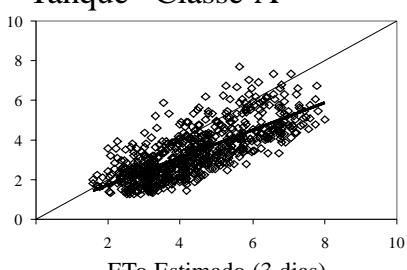

ETo Estimado (3 dias)
Jensen-Haise

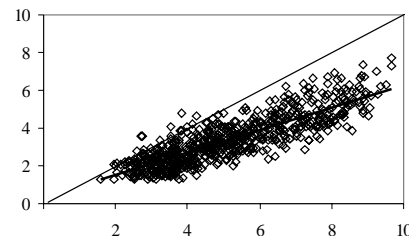

Atmômetro SEEI Modificado

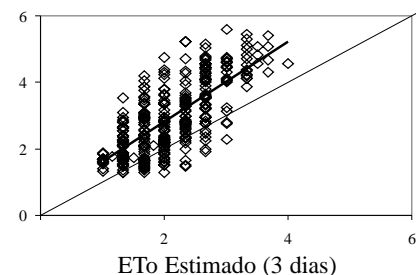

Figura 2. Correlações entre os valores da ETo medidos e estimados pelos métodos avaliados, em mm dia ${ }^{-1}$, no período de 3 dias, na região Norte Fluminense, $\mathrm{RJ}$

Penman Monteith-FAO

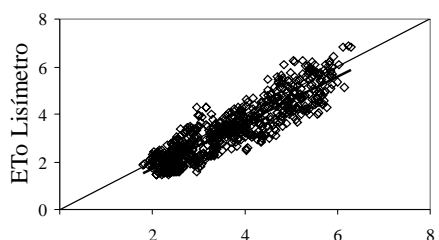

Hargreaves-Samani

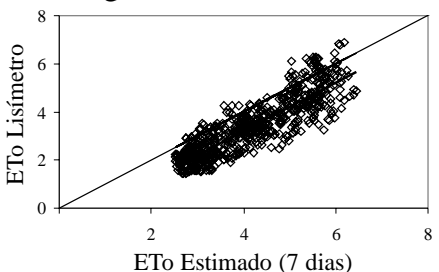

Makkink

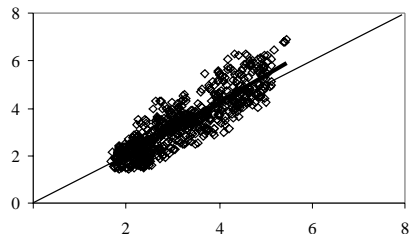

Radiação Solar-FAO

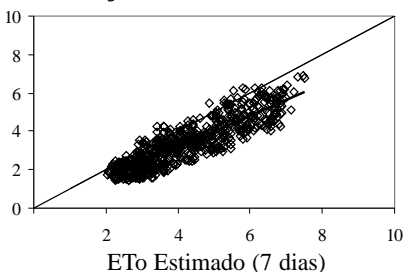

Linacre

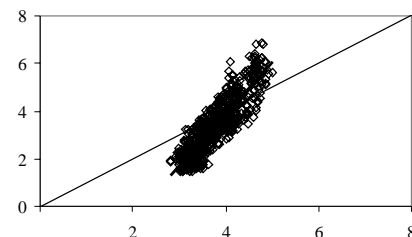

Tanque "Classe A"

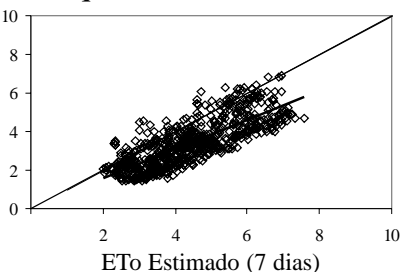

Jensen-Haise

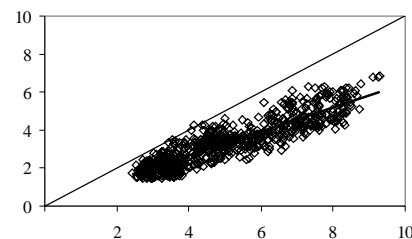

Atmômetro SEEI Modificado

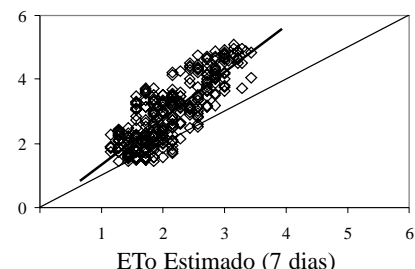

Figura 3. Correlações entre os valores da ETo medidos e estimados pelos métodos avaliados, em mm dia ${ }^{-1}$, no período de 7 dias, na região Norte Fluminense, RJ

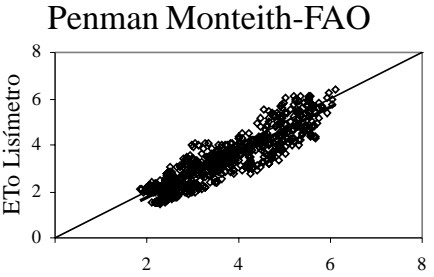

Hargreaves-Samani

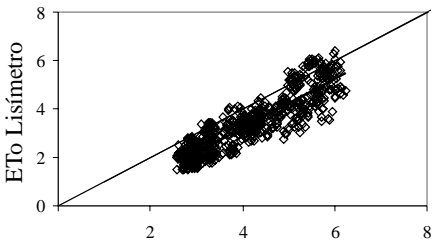

ETo Estimado (10 dias)
Makkink

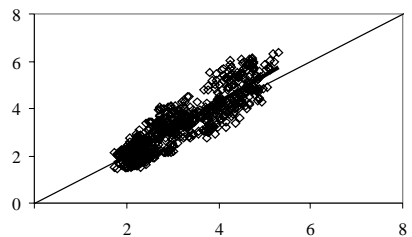

Radiação Solar-FAO

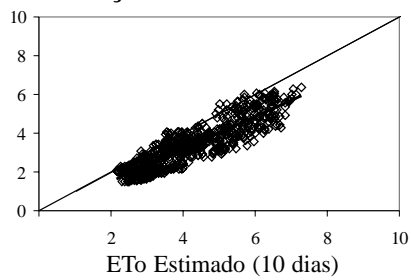

Linacre

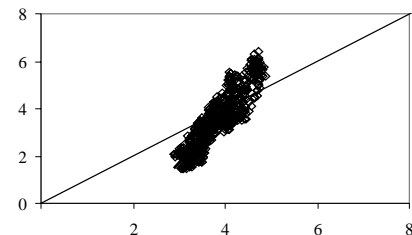

Tanque "Classe A"

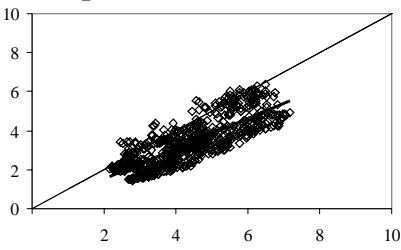

ETo Estimado (10 dias)
Jensen-Haise

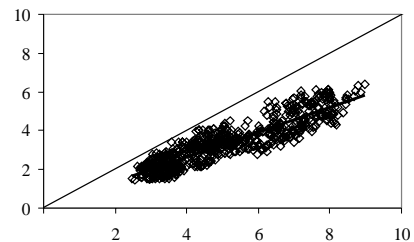

Atmômetro SEEI Modificado

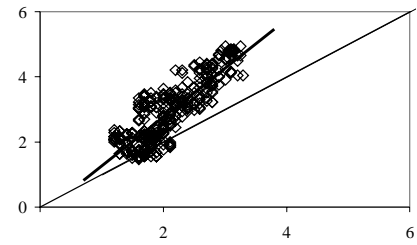

ETo Estimado (10 dias)

Figura 4. Correlações entre os valores da ETo medidos e estimados pelos métodos avaliados, em mm dia ${ }^{-1}$, no período de 10 dias, na região Norte Fluminense, RJ

aos outros métodos, com coeficientes lineares (b) apresentando valores próximos de 1 e os coeficientes angulares da reta valores próximos de zero. Silva et al. (1999) analisando o funcionamento de um lisímetro de pesagem com células de carga, na região de Piracicaba, SP, observaram que as mensurações da ETo medidas pelo lisímetro de pesagem apresentaram boa concordância com os valores estimados pelo método de Penman-Monteith, para um período de integração de 30 min ao longo de um dia, com coeficientes de determinação $\left(\mathrm{r}^{2}\right)$ variando de 0,69 a 0,80 .

O método de Hargreaves-Samani, apesar de apresentar, para período de 1 dia, um valor de $\mathrm{r}^{2}$ considerado baixo $(0,49)$, indicou 
coeficientes lineares bastante próximos do valor 1 para todos os períodos avaliados. Sendo este método simples e de fácil aplicação, por necessitar apenas de dados de temperatura máxima, temperatura mínima e a altitude local, a correlação satisfatória verificada com o lisímetro de pesagem o torna de uso potencial na região Norte Fluminense, RJ.

\section{CONCLUSÕES}

1. Todos os métodos avaliados, com exceção do Atmômetro SEEI modificado, atendem satisfatoriamente à estimativa da ETo na região norte fluminense, $\mathrm{RJ}$, principalmente em períodos de 7 e 10 dias.

2. Quando se dispõe somente de dados de temperatura máxima e mínima, o método de Hargreaves-Samani apresenta adequada precisão para se estimar a ETo na região, sobretudo para períodos superiores a 7 e 10 dias.

\section{AGRADECIMENTOS}

Os autores agradecem à Secretaria de Estado de Agricultura, Abastecimento, Pesca e Desenvolvimento do Interior (SEAAPI), à Fundação Estadual Norte Fluminense (FENORTE), à Fundação Carlos Chagas Filho de Amparo à Pesquisa do Estado do Rio de Janeiro (FAPERJ), à CAPES, ao CNPq e à
Empresa de Pesquisa Agropecuária do Estado do Rio de Janeiro (PESAGRO-Rio) pelo apoio financeiro e institucional, que permitiram a realização deste trabalho.

\section{LITERATURA CITADA}

Bernardo, S. Manual de Irrigação. 6 ed. Viçosa: UFV,1995. 657p. Bernardo, S.; Sousa, E.F.; Carvalho, J.A. Estimativa da evapotranspiração de referência (ETo), para as "áreas de baixada e de tabuleiros" da região Norte Fluminense. Campos dos Goytacazes: UENF, 1996. 14 p. Boletim Técnico

Doorenbos, J.; Pruitt, J.O. Crop water requirement. Rome: FAO, 1977. 144p. FAO Irrigation and Drainage Paper 24

Mello, J.L.P. Análise de sensibilidade dos componentes da equação de Penman-Monteith-FAO. Viçosa: UFV, 1998. 79p. Tese Doutorado

Pereira, A.R.; Villa Nova, N.A.; Sediyama, G.C. Evapo(transpi)ração. 1.ed. Piracicaba: FEALQ, 1997. 183p.

Peres, J.G.; Pereira, A.R.; Frizzone, J.A. Avaliação do modelo de Penman-Monteith para estimativa da evapotranspiração potencial de referência padronizada pela FAO. Revista Engenharia Rural, Piracicaba, v.6, n.1, p.65-77, 1995.

Silva F.C.; Folegatti, M.V.; Maggiotto, S.R. Análise do funcionamento de um lisímetro de pesagem com célula de carga. Revista Brasileira de Agrometeorologia , Santa Maria. v.7, n.1, 1999, p.53-58. 